\title{
Effect of classification based on combination of mutation and methylation in colorectal cancer prognosis
}

\author{
HARUHIKO AOYAGI ${ }^{1}$, SATORU IIDA ${ }^{1}$, HIROYUKI UETAKE ${ }^{2}$, TOSHIAKI ISHIKAWA ${ }^{2}$, \\ YOKO TAKAGI ${ }^{2}$, HIROTOSHI KOBAYASHI ${ }^{1}$, TETSURO HIGUCHI ${ }^{1}$, MASAMICHI YASUNO $^{1}$, \\ MASAYUKI ENOMOTO ${ }^{1}$ and KENICHI SUGIHARA ${ }^{1}$ \\ Departments of ${ }^{1}$ Surgical Oncology and ${ }^{2}$ Translational Oncology, Graduate School, \\ Tokyo Medical and Dental University, 1-5-45 Yushima, Bunkyo-ku, Tokyo 113-8519, Japan
}

Received August 23, 2010; Accepted November 3, 2010

DOI: $10.3892 /$ or.2010.1118

\begin{abstract}
Colorectal cancer (CRC) is caused by an accumulation of genetic alterations and epigenetic alterations. The molecular classification of CRCs based on genetic alterations and epigenetic alterations is evolving. Here, we examined mutations and methylation status in CRCs to determine if the combination of genetic and epigenetic alterations predicts prognosis. We examined 134 sporadic CRCs. We used the direct sequencing method to identify mutations in BRAF and AKT1, which are downstream of KRAS and PIK3CA, respectively, in the EGFR pathway. We used the Methylight method to determine the methylation status of hMLH1, p16, MINT1, MINT2 and MINT31. Both BRAF and AKT1 mutations were found in only one case $(0.75 \%)$. Aberrant methylation of hMLH1, p16, MINT1, MINT2 and MINT31 was detected in 22.4, 35.1, 32.8, 59.7 and $41.0 \%$ of cases, respectively. The clinicopathological factors were not significantly correlated to mutation or methylation. Among the patients who had no mutation in the EGFR pathway, the overall survival was significantly shorter in the patients with methylation compared to the patients with no methylation in hMLH1 and p16 $(\mathrm{p}=0.0318)$. Methylation could play a key role in the prognosis of patients without mutations in the EGFR pathway. The combination of genetic and epigenetic alterations may be a good marker for the prognosis of CRC patients.
\end{abstract}

\section{Introduction}

Colorectal cancer (CRC) is the third most common cancer in the world and the second most common cause of cancerrelated death (1). Of patients who undergo potentially curative

Correspondence to: Dr Satoru Iida, Department of Surgical Oncology, Graduate School, Tokyo Medical and Dental University, 1-5-45 Yushima, Bunkyo-ku, Tokyo 113-8519, Japan

E-mail: s-iida.srg2@tmd.ac.jp

Key words: mutation, methylation, colorectal cancer, prognosis surgery, $17 \%$ develop local recurrence or distant metastasis leading to a shorter survival time (2). Therefore, it is important to identify molecular markers of biological and prognostic significance and predictive value in patients with advanced CRC $(3,4)$.

CRC develops as a result of progressive accumulation of genetic alterations and epigenetic alterations (3-5). The elucidation of the human genome sequence (6) has showed that about 50-70 gene mutations are detected in CRC. Many studies have reported the importance of the mutations in the EGFR pathway, including the RAS/RAF pathway and the PI3K/AKT pathway (7-10). Gene mutations in the EGFR pathway are related to the efficiency of cetuximab or panitumumab therapy in metastatic CRC (11-14). The RAS/RAF pathway mediates the cellular response to extracellular signals that regulate cell growth, differentiation, and apoptosis (15). The PI3K/AKT pathway plays a central role in carcinogenesis since it is frequently activated and deregulated in the carcinogenic process of various human cancers (16). We previously examined the mutation of KRAS and PIK3CA in CRC patients and found that PIK3CA mutation is predictive of poor survival (17).

Gene methylation has been recognized as a third mechanism of Knudson's two-hit theory, and it is clear that methylation is associated with not only carcinogenesis but also the evolution and metastatic processes of cancer $(18,19)$. Epigenetic changes usually begin early in carcinogenesis, are potentially reversible, and can advance to gene alterations. Therefore, the detection of aberrant methylation is important for the early diagnosis, prognosis, and treatment of patients with CRC (20-22).

In the present study, we examined the mutation of BRAF and AKT1, which are downstream of KRAS and PIK3CA, respectively, and the methylation status of hMLH1, p16, MINT1, MINT2 and MINT31 to clarify whether the combination of genetic and epigenetic alterations might be used as parameters to predict prognosis in CRC.

\section{Materials and methods}

Patients and tissue samples. A total of 158 patients who had undergone surgical resection for primary sporadic colorectal 
cancer at the Department of Surgical Oncology, Tokyo Medical and Dental University (Tokyo, Japan), between March 2000 and April 2003 were targeted in our previous study. Of them, 134 patients, for whom genomic DNA was available were included in this study. This research was approved by the institutional review board of Tokyo Medical and Dental University, and written informed consent was obtained from all participants. The patients comprised 86 men and 48 women, ranging in age from 37 to 88 (mean, 64.5 years). Tumors were classified as proximal (proximal to the splenic flexure) or distal. There were 48 cancers in the proximal colon and 86 cancers in the distal colon, including the rectum. Histological classification and tumor staging were performed according to the International Union Against Cancer Tumor-Node-Metastasis (TNM) classification. No patient received preoperative chemotherapy or radiotherapy. After surgery, patients with stage III CRC received oral or intravenous 5-fluorouracil (5-FU)-based adjuvant chemotherapy, and patients with stage IV tumors received 5-FUbased systemic chemotherapy without any radiotherapy. Patients were prospectively followed-up after surgery for a median of 49 months. All resected specimens were fixed in $10 \% \mathrm{pH}$-neutral formalin and embedded in paraffin. In all cases, archival $H \& E$ slides of the primary tumors were retrieved and reviewed to confirm pathological features.

DNA extraction and mutation analysis. Tissue blocks were cut into $10-\mu \mathrm{m}$ thick sections with a microtome. The blade was changed and the microtome was cleaned after each specimen. After the specimens were deparaffinized and washed, tumor tissue was manually dissected with a razor blade in comparison to $\mathrm{H} \& \mathrm{E}$ slide. Tumor tissues were incubated overnight with proteinase $\mathrm{K}$ in digestion buffer, and then genomic DNA was extracted by a standard phenolchloroform method. Exon 1 of the KRAS gene, exons 9 and 20 of the PIK3CA gene, exon 15 of the BRAF gene, and exon 4 of the AKT1 gene were selected for mutation analysis, because mutations cluster in these regions. The exons were sequenced after PCR amplification. Primer sequences and PCR conditions are available upon request. PCR products were purified with Microcon YM-100 Centrifugal Filters (Millipore, MA) and Centri-Sep Columns (Princeton Separations, Adelphia, NJ) and then directly sequenced with a Big Dye Terminator Cycle Sequencing kit (3130 Genetic Analyser, Applied Biosystems, Foster City, CA).

Methylight analysis. Sodium bisulfite conversion and DNA recovery was performed using EpiTect Bisulfite (Qiagen). Following sodium bisulfite conversion, genomic DNA was analyzed by the Methylight technique, a fluorescence-based, real-time PCR (Q-PCR) assay (23) and the ABI Prism 7300 Real-Time PCR System (Taqman; Applied Biosystems). Six sets of primers and probes designed specifically for bisulfiteconverted DNA were used. One set was used to detect methylation in the gene of interest and the other five sets served as reference sets for $B$-actin (ACTB) to normalize for input DNA. The reference primers and probes were designed in a region of the ACTB gene that lacks CpG dinucleotides, thus allowing for equal amplification regardless of the methylation levels. Primer and probe sequences are available upon request. SssI- treated HCT-15 DNA was used as a fully methylated positive control (100\% methylation ratio). Parallel TaqMan PCR was performed with specific primers for the bisulfite-converted methylated sequence for a particular locus and with the ACTB reference primers. In each case, triplicate threshold cycle $(\mathrm{Ct})$ values were obtained and averaged, and expression levels were then evaluated by the $2-\Delta \Delta \mathrm{Ct}$ method (24). As an internal standard, each individual sample was normalized to its $B$-actin (ACTB) content and compared to the gene expression level of SssI-treated HCT-15 DNA (calibration sample) as follows: $2^{-\Delta \Delta \mathrm{Ct}}$, where $\Delta \Delta \mathrm{Ct}=(\mathrm{Ct}$-target-Ctreference) treated-sample - (Ct-target-Ct-reference) calibrator sample. We defined the percentage of fully methylated reference $(\mathrm{PMR})$ to be $2^{-\Delta \Delta \mathrm{Ct}} \times 100 \%$.

Statistical analysis. All statistical analyses were performed with StatView Software (version 5.0). To estimate differences between groups, the $\chi^{2}$ test, Fisher's exact test, Student's t-test and log-rank test were used as appropriate. The KaplanMeier method was used to estimate survival. Survival was calculated from the date of surgery. P-values $<0.05$ were considered to be significant.

\section{Results}

Mutation and methylation status in relation to clinicopathological parameters of 134 CRCs. KRAS mutations in exon 1 were found in $30.6 \%(41 / 134)$ of the cases, and PIK3CA mutations in exons 9 and 20 were found in $13.4 \%$ $(18 / 134)$ of the cases. Both BRAF and AKT1 mutations were found in only one case $(0.75 \%)$. One case with an AKT1 mutation also had a PIK3CA mutation, while one case with a BRAF mutation had no other mutations. There were no correlations between mutations in KRAS, BRAF, PIK3CA, and AKT1. There were no correlations between the RAS/RAF and PIK/AKT pathways. There were also no statistically significant differences between patients with mutations and patients without mutations in these pathways. The frequency of mutations is summarized in Table I.

Aberrant methylation of hMLH1, p16, MINT1, MINT2 and MINT31 was detected in 22.4\% (30/134), 35.1\% (47/134), $32.8 \%(44 / 134), 59.7 \%(80 / 134)$, and $41.0 \%(55 / 134)$, respectively. Aberrant methylation of p16 was significantly associated with tumor depth. The frequency of methylation is summarized in Table I.

Mutation or methylation status was not significantly correlated to the clinicopathological data (Tables I and II).

Relationship between the RAS/RAF and PIK/AKT pathways and methylation of $h M L H 1$ or p16 in CRCs. The relationship between the RAS/RAF and PIK/AKT pathways and methylation of hMLH1 or p16 is summarized in Table III. Although not statistically significant, hMLH1 methylation tended to be associated with p16 methylation $(\mathrm{p}=0.06)$.

Prognostic value of mutations in the RAS/RAF and PIK/AKT pathways and methylation of hMLH1 and p16 in CRCs. There was no statistically significant difference in overall survival between patients with and without a mutation in the RAF/RAF and PIK/AKT pathways ( $p=0.2436$; Fig. 1). Of 
Table I. Mutation status in relation to clinicopathological parameters of 134 CRC.

\begin{tabular}{|c|c|c|c|c|c|c|c|c|c|c|c|c|c|}
\hline & & \multicolumn{3}{|c|}{ KRAS } & \multicolumn{3}{|c|}{ PIK3CA } & \multicolumn{3}{|c|}{ BRAF } & \multicolumn{3}{|c|}{ AKT1 } \\
\hline & & $\mathrm{Wt}$ & Mut & P-value & $\mathrm{Wt}$ & Mut & P-value & $\mathrm{Wt}$ & Mut & $\mathrm{P}$-value & $\mathrm{Wt}$ & Mut & P-value \\
\hline No. of cases & 134 & 93 & 41 & & 116 & 18 & & 133 & 1 & & 133 & 1 & \\
\hline \multicolumn{14}{|l|}{ Gender } \\
\hline Male & $86(64.2)$ & 63 & 23 & 0.1952 & 75 & 11 & 0.7705 & 85 & 1 & 0.4533 & 86 & 0 & 0.1791 \\
\hline Female & $48(35.8)$ & 30 & 18 & & 41 & 7 & & 48 & 0 & & 47 & 1 & \\
\hline \multicolumn{14}{|l|}{ Tumor site } \\
\hline Proximal & $48(35.8)$ & 29 & 19 & 0.0917 & 39 & 9 & 0.1775 & 47 & 1 & 0.1791 & 48 & 0 & 0.4533 \\
\hline Distal & $86(64.2)$ & 64 & 22 & & 77 & 9 & & 86 & 0 & & 85 & 1 & \\
\hline \multicolumn{14}{|l|}{ Histology } \\
\hline Well & $46(34.3)$ & 32 & 14 & 0.9765 & 38 & 8 & 0.3313 & 46 & 0 & 0.468 & 45 & 1 & 0.165 \\
\hline Others & $88(65.7)$ & 61 & 27 & & 78 & 10 & & 87 & 1 & & 88 & 0 & \\
\hline \multicolumn{14}{|l|}{ pT } \\
\hline $\mathrm{T} 1, \mathrm{~T} 2$ & $22(16.4)$ & 17 & 5 & 0.3809 & 18 & 4 & 0.4749 & 22 & 0 & 0.6564 & 22 & 0 & 0.6564 \\
\hline $\mathrm{T} 3, \mathrm{~T} 4$ & $112(83.6)$ & 76 & 36 & & 98 & 14 & & 111 & 1 & & 111 & 1 & \\
\hline \multicolumn{14}{|l|}{$\mathrm{pN}$} \\
\hline Positive & $59(44.0)$ & 39 & 20 & 0.3809 & 52 & 7 & 0.8144 & 59 & 0 & 0.3661 & 59 & 0 & 0.3661 \\
\hline Negative & $75(56.0)$ & 54 & 21 & & 64 & 11 & & 74 & 1 & & 74 & 1 & \\
\hline \multicolumn{14}{|l|}{ TNM stage } \\
\hline I, II & $65(48.5)$ & 45 & 20 & 0.7391 & 57 & 8 & 0.7109 & 64 & 1 & 0.3011 & 64 & 1 & 0.3011 \\
\hline III, IV & $69(51.5)$ & 48 & 21 & & 59 & 10 & & 69 & 0 & & 69 & 0 & \\
\hline \multicolumn{14}{|l|}{$\begin{array}{l}\text { Lymphatic } \\
\text { invasion }\end{array}$} \\
\hline Positive & $101(75.4)$ & 69 & 32 & 0.6331 & 91 & 10 & 0.036 & 100 & 1 & 0.5661 & 100 & 1 & 0.5661 \\
\hline Negative & 33 (24.6) & 24 & 9 & & 25 & 8 & & 33 & 0 & & 33 & 0 & \\
\hline \multicolumn{14}{|l|}{$\begin{array}{l}\text { Venous } \\
\text { invasion }\end{array}$} \\
\hline Positive & $124(92.5)$ & 86 & 38 & 0.9666 & 111 & 13 & 0.0004 & 123 & 1 & 0.7756 & 123 & 1 & 0.7756 \\
\hline Negative & $10(7.5)$ & 7 & 3 & & 5 & 5 & & 10 & 0 & & 10 & 0 & \\
\hline
\end{tabular}

Wt, wild-type; Mut, mutated type.

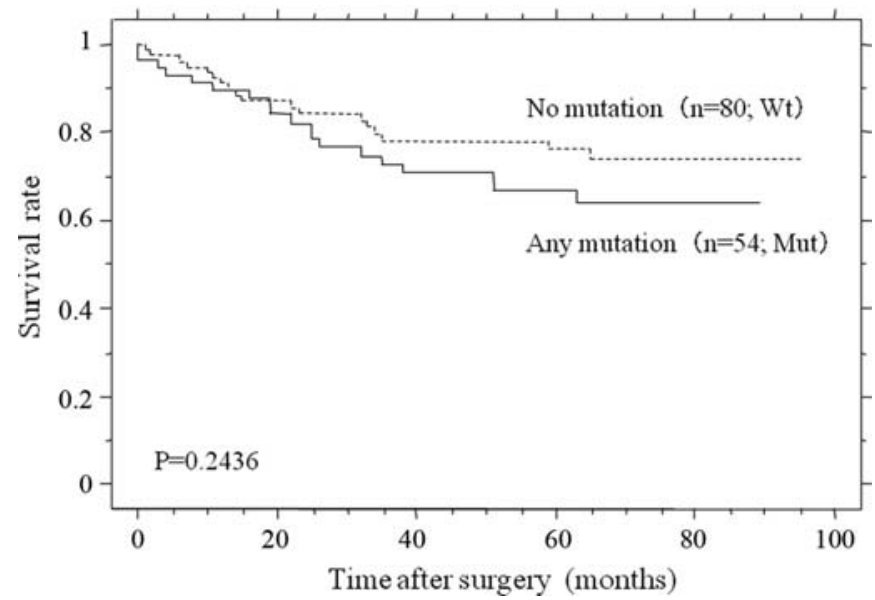

Figure 1. Overall survival in relation to mutation in the RAS/RAF and PIK/ AKT pathways.
134 patients with CRC, 54 had a mutation in the RAS/RAF or PIK/AKT pathway. Among these 54 patients, there was no significant difference in overall survival based on methylation of hMLH1 or p16 ( $\mathrm{p}=0.5463$; Fig. 2). Of the 134 patients with CRC, 80 had no mutations in the RAS/RAF and PIK/ AKT pathways. Among these wild-type patients, patients with methylated hMLH1 or p16 had a significantly shorter overall survival than those without methylation $(\mathrm{p}=0.0318$; Fig. 3).

\section{Discussion}

In the present study, we examined mutations in the RAS/RAF and PIK/AKT pathways and the methylation status of hMLH1, p16, MINT1, MINT2 and MINT31. We analyzed their correlations with clinicopathological factors and prognosis to determine whether these factors are novel prognostic markers 
Table II. Methylation status in relation to clinicopathological parameters of 134 CRC.

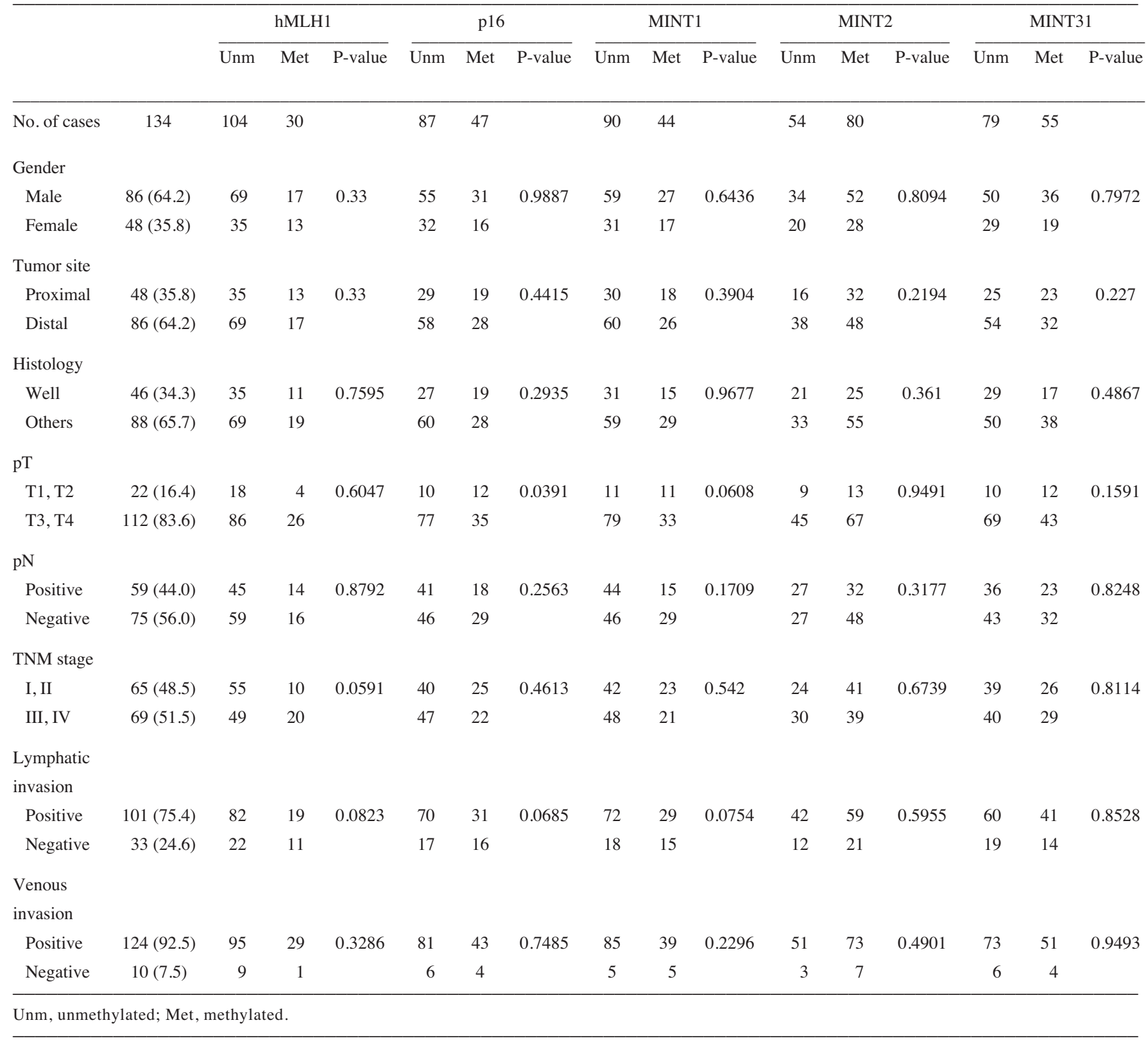

Table III. Relationship between mutation in EGFR pathway and methylation of hMLH1 and p16 in CRC.

\begin{tabular}{|c|c|c|c|c|c|c|c|c|c|}
\hline & \multicolumn{3}{|c|}{ PIK3CA } & \multicolumn{3}{|c|}{ p16 } & \multicolumn{3}{|c|}{ hMLH1 } \\
\hline & Mut & $\mathrm{Wt}$ & P-value & High & Low & $\mathrm{P}$-value & High & Low & P-value \\
\hline \multicolumn{10}{|l|}{$K R A S$} \\
\hline Mut & 6 & 35 & & 20 & 21 & & 8 & 33 & \\
\hline $\mathrm{Wt}$ & 12 & 81 & 0.787 & 27 & 65 & 0.03 & 22 & 71 & 0.596 \\
\hline \multicolumn{10}{|c|}{ PIK3CA } \\
\hline Mut & - & - & - & 6 & 12 & & 5 & 13 & \\
\hline $\mathrm{Wt}$ & - & - & - & 41 & 74 & 0.848 & 25 & 91 & 0.556 \\
\hline \multicolumn{10}{|l|}{ pl6 } \\
\hline High & - & - & - & - & - & - & 15 & 32 & \\
\hline Low & - & - & - & - & - & - & 15 & 71 & 0.06 \\
\hline
\end{tabular}

Wt, wild-type; Mut, mutated type. 


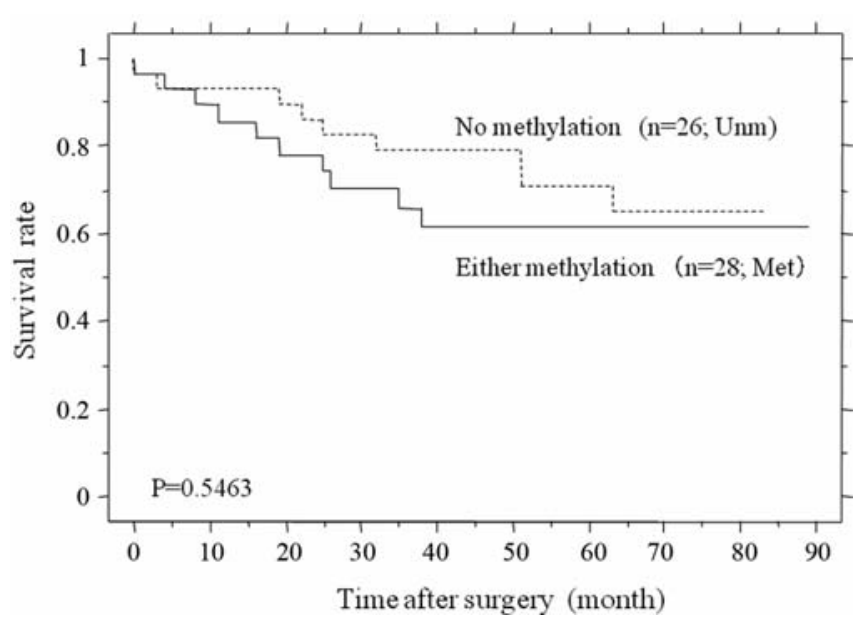

Figure 2. Overall survival in relation to hMLH1 and p16 methylation in the mutation group. Unm, unmethylated group; Met, methylated group.



Figure 3. Overall survival in relation to hMLH1 and p16 methylation in the wild-type group. Unm, unmethylated group; Met, methylated group.

of CRC. We found that the combination of mutation and methylation may be a good prognostic marker for CRC.

Mutations in the RAS/RAF and PIK/AKT pathways are present in CRC (7-10,25). We previously examined the mutation of KRAS and PIK3CA in CRC patients, and found that PIK3CA mutation is predictive of poor survival in these patients (17). In the present study, we examined the mutation status of BRAF and AKT1, which are downstream of KRAS and PIK3CA, respectively. BRAF and AKT1 mutations were detected in one case each. BRAF mutation has been reported to occur in about $15 \%$ of CRC cases, while V600E accounts for approximately $90 \%$ of the mutations $(26,27)$. The frequency of BRAF mutation in CRC patients differs among ethnic groups. Brim et al (28) analyzed BRAF mutation in CRC patients of different ethnic groups, African American, Omani and Iranian. Among these CRC patients, BRAF mutation was detected in $10 \%$ of the African Americans, $19 \%$ of the Omanis, and $2 \%$ of the Iranians. The frequency of BRAF mutation in Asia tends to be low, reported at approximately 5\% (29-32). These differences among ethnic groups may be due to different lifestyle factors such as diet, alcohol and smoking $(33,34)$. Our study indicates that the frequency of BRAF mutations in Asian patients with CRC is lower than in other ethnic groups. BRAF mutation in CRC is associated with microsatellite instability-high colorectal cancer (MSI-H CRC) $(9,10,15,27)$. MSI-H CRC is often detected in the early stage of cancers. Most samples in the present study were from CRC patients in an advanced stage, so it is possible that the frequency of BRAF mutation was low. Carpen et al (35) found AKT1 mutation in $6 \%$ of CRC patients, but other studies have reported smaller frequencies of AKT1 mutation. Kim et al (36) found no AKT1 mutations in 104 CRC patients. In a study of 88 CRC patients, Bleeker et al (37) found only one case with an AKT1 mutation. Therefore, it is possible that the frequency of AKT1 mutation in CRC cases is lower than the $6 \%$ reported by Carpen et al. In the present study of CRC, the frequency of BRAF and AKT1 mutations was less than the frequency of KRAS and PIK3CA mutations; therefore, it is possible that the mutation of KRAS and PIK3CA is more important than the mutation of BRAF and AKT1 in carcinogenesis of CRC.

We examined the methylation status of five genes, but found no significant correlation between methylation status and clinicopathological factors. Many reports have found a relationship between methylation of these genes and CRC (38-40). The hMLH1 gene is methylated in MSI-H CRC, and the relationship between methylated hMLH1 and CRC prognosis has been discussed in many studies (41-43). Wettergren et al (44) reported that p16 hypermethylation may be a prognostic marker in CRC patients. Therefore, we focused on methylation of these two genes, hMLH1 and p16.

The combination of genetic alterations and epigenetic alterations may provide a good marker for the prognosis of CRC patients. Shen et al (45) analyzed both mutation and methylation in primary $\mathrm{CRC}$ and found that $\mathrm{CRC}$ consists of three distinct subclasses, each of which is fairly homogeneous. Lee et al (29) divided CRC patients into four groups based on classification of the RAS/RAF mutation and CIMP, and showed that this classification may be a very effective prognostic marker. Similarly, Ogino et al (46) showed that patients with CIMP-low and mutated BRAF have a shorter survival than those with other CIMP/BRAF types.

In the present study, overall survival was not associated with mutations in the RAS/RAF and PIK/AKT pathways. Thus, genetic classification was not useful as a prognostic marker among these patients. Overall survival of patients with mutations was not associated with the methylation status of hMLH1 and p16. However, among the patients without mutations, overall survival was significantly shorter in patients with any methylation than in those without methylation $(p=0.0318)$. Thus, the combination of genetic and epigenetic classification has potential as a good prognostic marker among CRC patients. One possible reason for the lack of prognostic significance of epigenetic and genetic parameters among patients with mutations is that the genetic alterations may predominate in carcinogenesis of CRC; therefore, it is reasonable that the overall survival of wild-type patients is significantly shorter in when methylation occurs compared to no methylation; that is, methylation may play a central role in carcinogenesis of wild-type CRC. Thus, the combination 
of genetic and epigenetic alterations may be used as a good marker for prognosis in CRC patients.

In conclusion, we found that genetic alteration by itself was not significantly associated with prognosis; however, the combination of genetic alteration and epigenetic alteration may be a good marker for the prognosis of CRC.

\section{References}

1. Ricchi P, Zaeeilli R, Di Palma A and Acquaviva AM: Nonsteroidal anti-inflammatory drugs in colorectal cancer: from prevention to therapy. Br J Cancer 88: 803-807, 2003.

2. Kobayashi H, Mochizuki H, Sugihara K, et al: Characteristics of recurrence and surveillance tools after curative resection for colorectal cancer: a multicenter study. Surgery 141: 67-75, 2007.

3. Kinzler KW and Vogelstein B: Lessons from hereditary colorectal cancer. Cell 87: 159-170, 1996.

4. Jass JR: Classification of colorectal cancer based on correlation of clinical, morphological and molecular features. Histopathology 50: 113-130, 2007.

5. Ogino S and Goel A: Molecular classification and correlates in colorectal cancer. J Mol Diagn 10: 13-27, 2008.

6. Sjoblom T, Jones S, Wood LD, et al: The consensus coding sequences of human breast and colorectal cancers. Science 314: 268-274, 2006.

7. Nosho K, Kawasaki T, Ohnishi M, et al: PIK3CA mutation in colorectal cancer: relationship with genetic and epigenetic alterations. Neoplasia 10: 534-541, 2008.

8. Nagasaka T, Koi M, Kloor M, et al: Mutations in both KRAS and BRAF may contribute to methylator phenotype in colon cancer. Gastroenterology 134: 1950-1960, 2008

9. Samowitz WS, Sweeney C, Herrick J, et al: Poor survival associated with the BRAF V600E mutation in microsatellitestable colon cancers. Cancer Res 65: 6063-6070, 2005.

10. Oliviera C, Velho S, Moutinho C, et al: KRAS and BRAF oncogenic mutations in MSS colorectal carcinoma progression. Oncogene 26: 158-163, 2007.

11. Moroni M, Veronese S, Benvenuti S, et al: Gene copy number for epidermal growth factor receptor (EGFR) and clinical response to antiEGFR treatment in colorectal cancer: a cohort study. Lancet Oncol 6: 279-286, 2005

12. Lievre A, Bachet JB, Le Corre D, et al: KRAS mutation status is predictive of response to cetuximab therapy in colorectal cancer. Cancer Res 66: 3992-3995, 2006.

13. Cunningham D and Wong R: Using predictive biomarkers to select patients with advanced colorectal cancer for treatment with epidermal growth factor receptor antibodies. J Clin Oncol 26: 5668-5670, 2008.

14. Seth R, Crook S, Ibrahem S, et al: Concomitant mutations and splice variants in KRAS and BRAF demonstrate complex perturbation of the RAS/RAF signaling pathway in advanced colorectal cancer. Gut 58: 1234-1241, 2009.

15. Tanaka H, Deng G, Matsuzaki K, et al: BRAF mutation, $\mathrm{CpG}$ island methylator phenotype and microsatellite instability occur more frequently and concordantly in mucinous than nonmucinous colorectal cancer. Int J Cancer 118: 2765-2771, 2006.

16. Oikonomou E and Pintzas A: Cancer genetics of sporadic colorectal cancer: BRAF and PIK3CA mutations, their impact on signaling and novel targeted therapies. Anticancer Res 26: 1077-1084, 2006.

17. Kato S, Iida S, Higchi T, et al: PIK3CA mutation is predictive of poor survival in patients with colorectal cancer. Int J Cancer 121: 1771-1778, 2007

18. Feinberg AP and Tycko B: The history of cancer epigenetics. Nat Rev Cancer 4: 143-153, 2004

19. Jones PA and Baylin SB: The fundamental role of epigenetic events in cancer. Nat Rev Genet 3: 415-428, 2002.

20. Herman JG and Baylin SB: Gene silencing in cancer in association with promoter hypermethylation. N Engl J Med 349: 2042-2054, 2003

21. Baylin SB, Esteller M, Rountree MR, Bachman KE, Schuebel K and Herman JG: Aberrant patterns of DNA methylation, chromatin formation and gene expression in cancer. Hum Mol Genet 10: 687-692, 2001

22. Verma M and Strivastava S: Epigenetics in cancer: implications for early detection and prevention. Lancet Oncol 3: 755-763, 2002.
23. Eads CA, Danenberg KD, Kawakami K, et al: MethyLight: a high-throughput assay to measure DNA methylation. Nucleic Acids Res 28: E32, 2000

24. Shirota Y, Stoehlmacher J, Brabender J, et al: ERCC1 and thymidylate synthase mRNA levels predict survival for colorectal cancer patients receiving combination oxaliplatin and fluorouracil chemotherapy. J Clin Oncol 19: 4298-4304, 2001.

25. Barault L, Veyrie N, Jooste V, et al: Mutations in the RASMAPK, PI(3)K (phosphatidylinositol-3-OH kinase) signaling network correlate with poor survival in a population-based series of colon cancers. Int J Cancer 122: 2255-2259, 2008.

26. Davies H, Bingnell GR, Cox C, et al: Mutations of the BRAF gene in human cancer. Nature 417: 949-954, 2002.

27. Rajagopalan H, Bardelli A, Lengauer C, Kinzler KW, Vogelstein B and Velculescu VE: Tumorigenesis: RAF/RAS oncogenes and mismatch-repair status. Nature 418: 934, 2002.

28. Brim H, Mokarram P, Naghibalhossaini F, et al: Impact on BRAF, MLH1 on the incidence of microsatellite instability high colorectal cancer in population based study. Mol Cancer 7: 68, 2008.

29. Lee S, Cho NY, Choi M, Yoo EJ, Kim JH and Kang GH: Clinicopathological features of $\mathrm{CpG}$ island methylator phenotypepositive colorectal cancer and its adverse prognosis in relation to KRAS/BRAF mutation. Pathol Int 58: 104-113, 2008.

30. Chang SC, Lin JK, Yang SH, Wang HS, Li AFY and Chi CW: Relationship between genetic alterations and prognosis in sporadic colorectal cancer. Int J Cancer 118: 1721-1727, 2008.

31. Yuen ST, Davis H, Chan TL, et al: Similarity of the phenotypic patterns associated with BRAF and KRAS mutations in colorectal neoplasia. Cancer Res 62: 6451-6455, 2002.

32. Vilkin A, Niv Y, Nagasaka T, et al: Microsatellite instability, MLH1 promoter methylation, and BRAF mutation analysis in sporadic colorectal cancers of different ethnic groups in Israel. Cancer 115: 760-769, 2009 .

33. Slattery ML, Curtin K, Levin TR, et al: Diet and lifestyle factor associations with $\mathrm{CpG}$ island methylator phenotype and BRAF mutations in colon cancer. Int J Cancer 120: 656-663, 2006.

34. Fransen K, Klintenas M, Osterstrom A, Dimberg J, Monstein HJ and Soderkvist P: Mutation analysis of the BRAF, ARAF and RAF-1 genes in human colorectal adenocarcinomas. Carcinogenesis 25: 527-533, 2004.

35. Carpen JD, Faber AL, Horn C, et al: A transforming mutation in the pleckstrin homology domain of AKT1 in cancer. Nature 448: 439-445, 2007.

36. Kim MS, Jeong EG and Lee SH: Mutational analysis of oncogenic AKT E17K mutation in common solid cancers and acute leukaemias. Br J Cancer 98: 1533-1535, 2008.

37. Bleeker FE, Felicioni L, Buttitta F, et al: AKT1E17K in human solid tumours. Oncogene 27: 5648-5650, 2008.

38. Toyota M, Ahuja N, Ohe-Toyota M, Baylin SB and Issa JP: $\mathrm{CpG}$ island methylator phenotype in colorectal cancer. Proc Natl Acad Sci USA 96: 8681-8686, 1999.

39. Goel A, Nagasaka T, Arnold CN, et al: The CpG island methylator phenotype and chromosomal instability are inversely correlated in sporadic colorectal cancer. Gastroenterology 132: 127-138, 2007.

40. Ogino S, Kawasaki T, Kirkner GJ, Kraft P, Loda M and Fuchs CS: Evaluation of markers for $\mathrm{CpG}$ island methylator phenotype (CIMP) in colorectal cancer by a large population-based sample. J Mol Diagn 9: 305-314, 2007.

41. Shannon BA and Lacopetta BJ: Methylation of the hMLH1, p16 and MDR1 genes in colorectal carcinoma: associations with clinicopathological features. Cancer Lett 167: 91-97, 2001.

42. Herman JG, Umar A, Polyak K, et al: Incidence and functional consequences of hMLH1 promoter hypermethylation in colorectal carcinoma. Proc Natl Acad Sci USA 95: 6870-6875, 1998.

43. Veigl ML, Kasturi L, Olechnowicz J, et al: Biallelic inactivation of hMLH1 by epigenetic gene silencing, a novel mechanism causing human MSI cancers. Proc Natl Acad Sci USA 95: 8698-8702, 1998.

44. Wettergren Y, Odin E, Nilsson S, Carlsson G and Gustavsson B: p16INK4a gene promoter hypermethylation in mucosa as a prognostic factor for patients with colorectal cancer. Int Mol Med 14: 412-421, 2008

45. Shen L, Toyota M, Kondo Y, et al: Integrated genetic and epigenetic analysis identifies three different subclass of colon cancer. Proc Natl Acad Sci USA 104: 18654-18659, 2007.

46. Ogino S, Nosho K, Kirkner GJ, et al: $\mathrm{CpG}$ island methylator phenotype, microsatellite instability, BRAF mutation and clinical outcome in colon cancer. Gut 58: 90-96, 2009. 